\title{
Efficacy of nab-paclitaxel plus trastuzumab in a long-surviving heavily pretreated HER2-positive breast cancer patient with brain metastases
}

This article was published in the following Dove Press journal:

OncoTargets and Therapy

2 February 2015

Number of times this article has been viewed

\author{
Giuseppina Rosaria Rita \\ Ricciardi ${ }^{1,2}$ \\ Alessandro Russo ${ }^{1,2}$ \\ Tindara Franchina ${ }^{1,2}$ \\ Giuseppa Ferraro',2 \\ Vincenzo Adamo ${ }^{1,2}$ \\ 'Department of Human Pathology, \\ University of Messina, Messina, Italy; \\ ${ }^{2}$ Medical Oncology Unit, Azienda \\ Ospedaliera Ospedali Riuniti Papardo- \\ Piemonte, Messina, Italy
}

Correspondence: Vincenzo Adamo UOC Oncologia Medica, Azienda Ospedaliera Ospedali Riuniti PapardoPiemonte, Contrada Papardo, 98I58

Messina, Italy

Tel +390903996150

Email vadamo@unime.it
Abstract: Brain metastases (BMs) represent a major issue in clinical practice and are associated with a significant worsening of patient's quality of life and, often, a dismal prognosis. Breast cancer $(\mathrm{BC})$ is the second most common solid malignancy that metastasizes to the central nervous system. Incidence of BM varies according to the tumor subtype, with higher rates in patients with epidermal growth factor receptor 2 (HER2) overexpression and in triple negative breast cancers. The incidence of BM in HER2-positive BC patients has increased as a consequence of the success of trastuzumab-based therapy. BM represents an emerging unmet medical need and no specific treatment options exist because, until recently, nearly all randomized clinical trials in metastatic breast cancer (MBC) excluded such patients. Therefore, novel approaches in this setting are eagerly awaited. Herein, we report a lengthy progression-free survival with the combination trastuzumab/nanoparticle albumin-bound (nab)-paclitaxel in a heavily pretreated HER2-positive BC patient with BM. The long-term disease stabilization reported in the present case ( $>13$ months) is of note for several reasons. First, the nab-paclitaxel plus trastuzumab combination, despite several previous lines of treatment, some of which were associated with known activity on BM, was the first systemic therapy not associated with central nervous system recurrence, avoiding recourse to additional locoregional treatments. Moreover, this combination was associated with long extracranial stabilization with minimal toxicity. The remarkably lengthy progression-free survival reported in our case with the nab-paclitaxel plus trastuzumab combination further confirms the efficacy and the favorable toxicity profile of this promising schedule that showed intriguing results in two phase II studies in first-line MBC and suggests a possible activity on BM. In conclusion, weekly nab-paclitaxel plus trastuzumab may represent a valuable option in the treatment of HER2-positive MBC with BM after radiotherapy and is effective and associated with a good toxicity profile, even in heavily pretreated patients.

Keywords: HER-2, breast cancer, nab-paclitaxel, brain metastases, trastuzumab, long survivor

\section{Background}

Brain metastases (BMs) represent a major issue in clinical practice and are associated with a significant worsening of a patient's quality of life and, often, a dismal prognosis.

Breast cancer $(\mathrm{BC})$ is the second most common solid malignancy that metastasizes to the central nervous system (CNS), with an estimated incidence of $10 \%-16 \%$ during the course of the disease, reaching up to $30 \%$ on autopsy. ${ }^{1,2}$

The incidence of BM varies according to the tumor subtype, with higher rates in patients with overexpression of the epidermal growth factor receptor 2 (HER2) and in triple negative $\mathrm{BC}$ patients. The higher tendency to CNS metastatic spread among HER2-positive BC patients is largely unknown, but it is likely multifactorial: inherent 
biologic tropism for CNS of this molecular subtype, a more aggressive nature, and the ability to produce protumorigenic and prometastatic enzymes. In addition, it has been hypothesized that the incidence of BM in HER2-positive BCs has increased as a consequence of the success of trastuzumabbased therapy. Because trastuzumab does not cross the blood-brain barrier (BBB) due to its high molecular weight $(180 \mathrm{kDa})$, the growing incidence of BM among patients with metastatic breast cancer (MBC) treated with trastuzumab, especially in the setting of controlled extracranial disease, has been attributed to the more frequent diagnosis of CNS involvement that would be otherwise clinically silent before death, due to the aggressive course of the disease in the pretrastuzumab era. ${ }^{1-3}$ Moreover, three distinct meta-analyses of the use of trastuzumab in the adjuvant setting reported a higher incidence of $\mathrm{CNS}$ recurrence and an increased risk of BM as first metastatic site among HER2-positive BC patients. ${ }^{4-6}$

BMs represent an emerging unmet medical need and no specific treatment options exist because, until recently, nearly all randomized clinical trials in MBC excluded such patients. Therefore, novel approaches in this setting are eagerly awaited.

Herein, we report a lengthy progression-free survival (PFS) with the combination trastuzumab/nanoparticle albumin-bound (nab)-paclitaxel in a heavily pretreated HER2-positive MBC patient with BMs.

\section{Case presentation}

A 31-year-old woman underwent radical left mastectomy plus ipsilateral axillary lymphadenectomy in April 2004 because of a poorly differentiated ductal carcinoma, pT2 N3(16/23) M0. The pathology review revealed a luminal B HER2-positive BC estrogen receptor (ER): 70\%; progesterone receptor (PgR): 40\%; ki-67>25\%; HER2: 3+ detected by immunohistochemistry. On the basis of the biological assessment, the tumor histotype, and the disease stage, she underwent adjuvant chemotherapy with fluouracil, epirubicin, and cyclophosphamide $\left(\mathrm{FEC}_{(90)}\right)$ for six cycles, followed by locoregional radiotherapy and hormonotherapy with luteinizing hormone-releasing hormone (LHRH) analog + tamoxifen. No adjuvant trastuzumab therapy was administered because of regulatory issues.

In January 2009, follow-up imaging revealed a recurrence in the lung and then she was put on first-line metastatic therapy docetaxel ( $75 \mathrm{mg} / \mathrm{m}^{2}$ of body surface area every 21 days) plus 3-weekly trastuzumab (with a loading dose of $8 \mathrm{mg} / \mathrm{kg}$, followed by a maintenance dose of $6 \mathrm{mg} / \mathrm{kg}$ ) for 8 months. No significant cardiac adverse events were reported.
In October 2009, there was a sudden onset of headache and dizziness. Then, a brain magnetic resonance imaging (MRI) scan was performed, documenting multiple contrast-enhancing lesions disseminated in the capsular left nucleus, in the left temporal lobe, and in the right cerebellar cortical region. The metastatic spread to the brain occurred in the context of a controlled extracranial disease. On the basis of the brain progressive disease and the substantial stability of the lung lesions, in November 2009, she received brain stereotactic radiosurgery (SRS) with Cyberknife ${ }^{\circledR}$ system (Accuray Inc, Sunnyvale, CA, USA), followed by whole-brain radiotherapy, with rapid relief of the neurological symptoms. Then, trastuzumab-based therapy was continued, with stable disease.

In May 2010, a positron emission tomography-computed tomography (PET/CT) scan confirmed stability of the lung lesions, but a brain MRI revealed further progressive disease with a novel lesion in the left cerebellar tonsil, treated with SRS with Cyberknife ${ }^{\circledR}$ system. After SRS, she continued trastuzumab-based therapy beyond CNS progression.

In February 2011, a novel brain MRI scan showed CNS progressive disease, with two additional lesions in the right temporal lobe and in the right parietal lobe and recurrence of previously treated left temporal lesion, despite stable extracranial lesion. A novel SRS on the three metastatic CNS lesions was performed. Then, trastuzumab-based therapy was discontinued and in March 2011, she began treatment with lapatinib $1,250 \mathrm{mg}$ daily plus capecitabine $1,000 \mathrm{mg} / \mathrm{m}^{2}$ twice daily on days $1-14$ every 21 days, with a CNS partial response as a maximum response. After a PFS of 9 months, she experienced bone progressive disease in the pelvis. Thus, lapatinib therapy was switched to weekly trastuzumab plus vinorelbine (at a dose of $25 \mathrm{mg} / \mathrm{m}^{2}$ of body surface area once a week), which led to disease stabilization.

A CT scan in September 2012 showed extensive progressive disease with appearance of new brain, liver, bone, and lung metastases (Figure 1). A novel SRS treatment was performed on the two novel lesions in the left frontal lobe and in the right cerebellar hemisphere and, then in November 2012, it was decided to administer a novel therapeutic line with weekly nab-paclitaxel at a dose of $125 \mathrm{mg} / \mathrm{m}^{2}$, continuing weekly trastuzumab beyond progression. Four courses of nabpaclitaxel/trastuzumab therapy were delivered, with evidence of a partial response in the lung lesion, with substantial stable disease (SD) in the liver and bone metastases and a minimal response in the brain nodules (Figure 2). Hence, she continued treatment for up to 14 cycles with the same schedule with substantial stable disease (Figure 3) and minimal neurological treatment-related toxicities. A complete list of all the patient's 

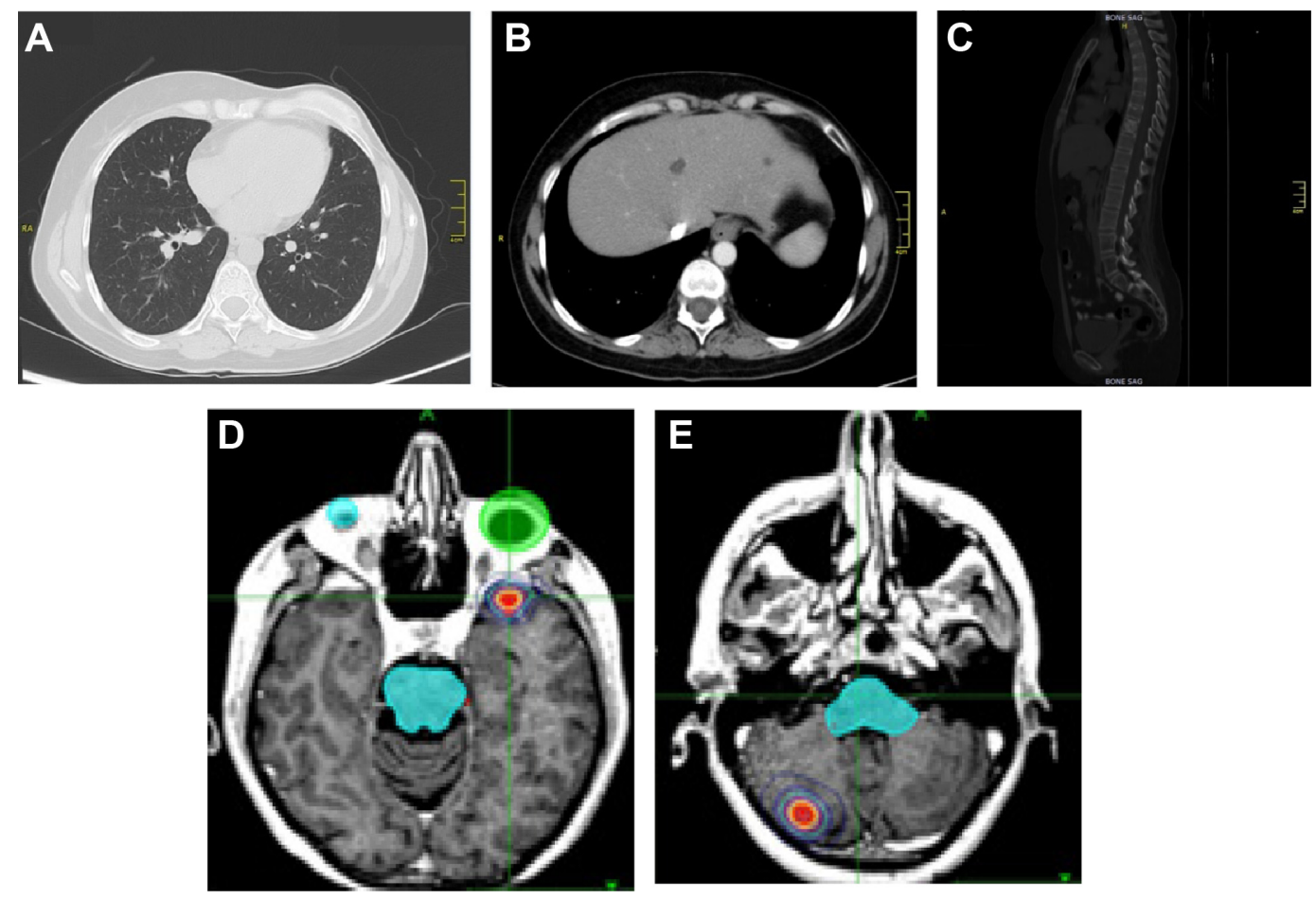

Figure I Lung (A), liver (B), bone (C), and brain (D, E) metastases before start of nab-paclitaxel/trastuzumab treatment. Abbreviation: nab, nanoparticle albumin-bound.

treatments is reported in Figure 4. We performed a cardiological evaluation every 3 months with echocardiography, showing no cardiac dysfunction. Therefore, this combination was associated with good cardiac toxicity profile. After 13 months
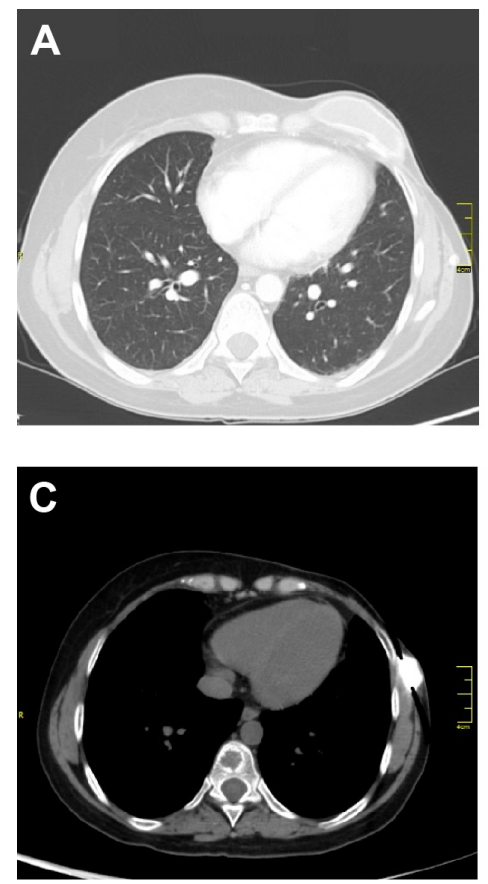

of substantial stable disease, she experienced further disease progression, with increase in the number and dimension of liver, brain, and pulmonary metastases, and a novel treatment was started.
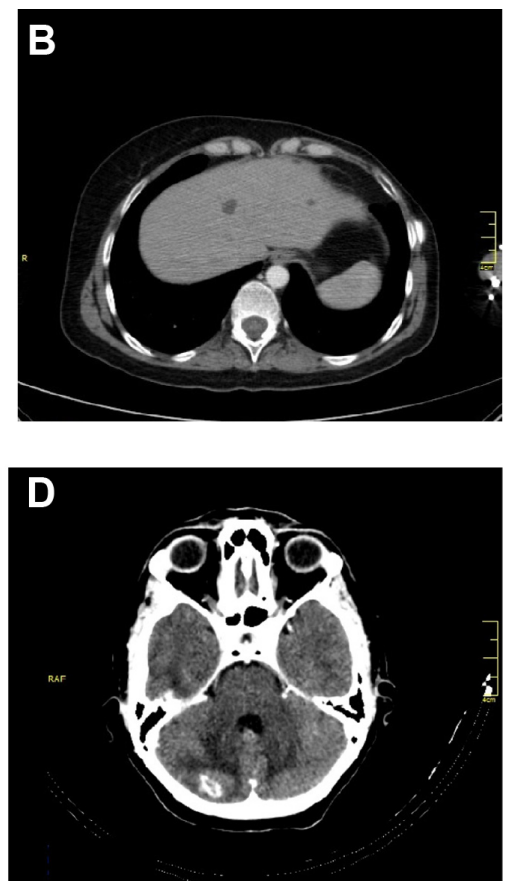

Figure 2 Lung (A), Liver (B), Bone (C) and Brain (D) lesions after four courses of nab-paclitaxel/trastuzumab combination. Abbreviation: nab, nanoparticle albumin-bound. 

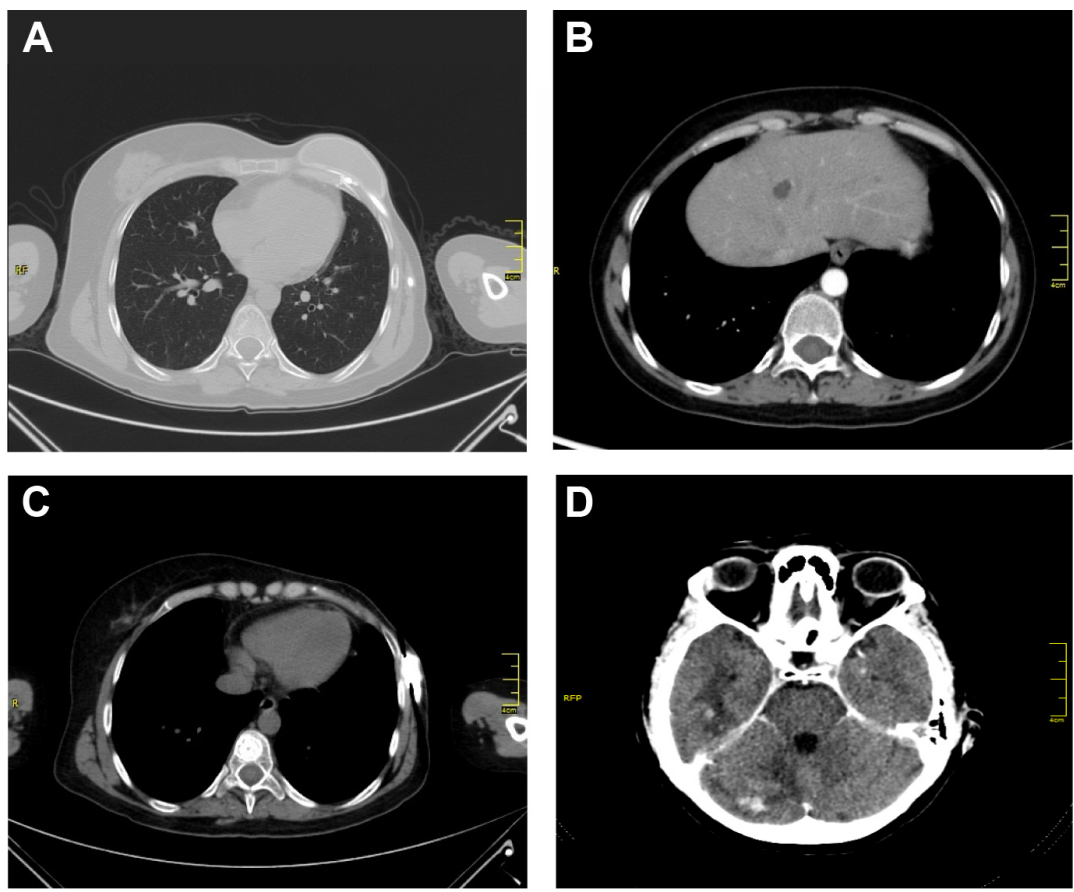

Figure 3 Lung (A), Liver (B), Bone (C) and Brain (D) lesions after nine courses of nab-paclitaxel/trastuzumab combination.

Abbreviation: nab, nanoparticle albumin-bound.

\section{Discussion}

$\mathrm{BC}$ is the second most common solid malignancy that metastasizes to the CNS, with an estimated incidence of $10 \%-16 \%$ during the course of the disease, reaching up to $30 \%$ on autopsy; however, the incidence of BM varies according to the tumor subtype, with higher rates in patients with HER2positive and triple-negative BCs. ${ }^{1,2}$
Emergence of brain metastases represents one of the most life-threatening conditions in patients with HER2-positive MBC and is classically associated with a poor prognosis. Locoregional treatments, such as radiotherapy (either as whole-brain radiotherapy or as SRS), surgery or a combination of these therapies, are generally offered either for symptom palliation or with curative intent and are associated with small survival improvements.

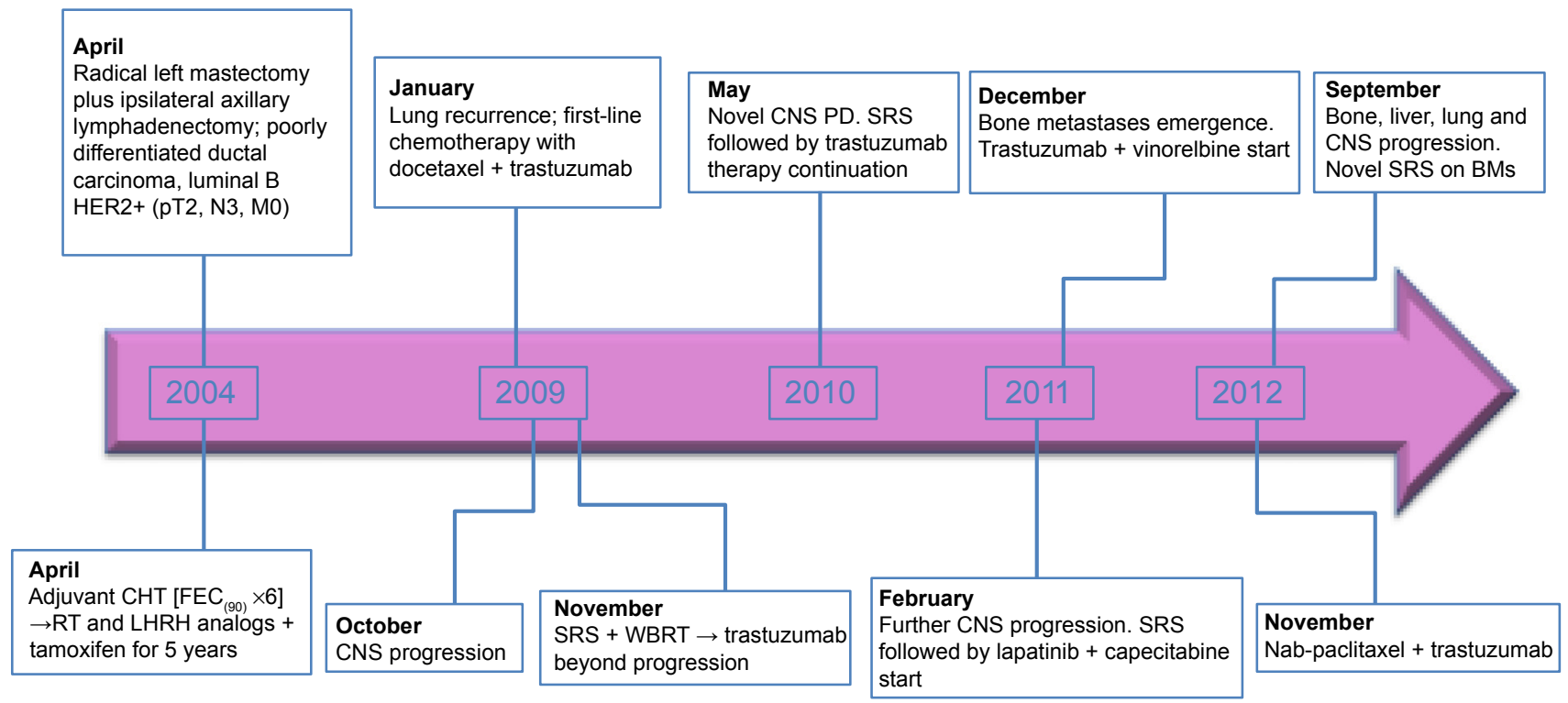

Figure 4 Timeline of patient's diagnosis and treatments.

Abbreviations: BMs, brain metastasis; CHT, chemotherapy; CNS, central nervous system; FEC, fluouracil, epirubicin, and cyclophosphamide; HER, human epidermal growth factor receptor; PD, progressive disease; SRS, stereotactic radiosurgery; nab, nanoparticle albumin-bound; LHRH, luteinizing hormone-releasing hormone; RT, radiotherapy; WBRT, whole-brain radiotherapy. 
Traditionally, systemic treatments were considered mostly ineffective on BM, with only a handful of clinical responses to most standard cytotoxic drugs. These disappointing results may reflect the intrinsic increased resistance of BM, which often emerges late in the course of disease, compared with other systemic metastases, as a consequence of accumulated mutations in the metastatic tumor cells after multiple rounds of previous chemotherapies. In addition, the presence of the $\mathrm{BBB}$, a multicellular vascular structure that separates the CNS from the peripheral blood circulation, prohibits an adequate brain penetration to most of the standard systemic anticancer agents, limiting their clinical efficacy, because of their chemical structure/dimensions and/or the removal by drug efflux pumps, such as the P-glycoprotein. ${ }^{7,8}$ Brain metastases may alter the integrity of the $\mathrm{BBB}$, increasing its permeability compared with that in a normal brain. However, preclinical studies support the notion that this increased permeability could not be homogeneous, with only a small subset of BM $(\sim 10 \%)$ having sufficient permeability to show a response to common cytotoxic agents, such as paclitaxel. ${ }^{9}$ Interestingly, in a small retrospective study, a significant correlation between BC subtype and BBB disruption was reported, with HER2positive $\mathrm{BC}$ tending to preserve $\mathrm{BBB}$ integrity compared with triple-negative and basal-like BCs. ${ }^{10}$ In addition, the BBB permeability may be altered in patients with $\mathrm{BM}$ because of other perturbing factors such as radiotherapy too. ${ }^{11}$

Trastuzumab-based therapy is firmly established as the standard of care for patients with HER2-positive BC, demonstrating survival benefits in large, randomized clinical trials in both EBC. ${ }^{12-14}$ Despite this impressive clinical activity, concern has been expressed that trastuzumab therapy may be associated with an increased frequency of CNS relapse. ${ }^{4-6}$ However, retrospective studies reported that patients with HER2-overexpressing MBC who received trastuzumab after diagnosis of BM survive longer, suggesting that continuation of trastuzumab-based therapy beyond CNS progression, as shown in the present case, in patients with good performance status is beneficial in terms of survival. ${ }^{15-17}$ From these studies, however, it is difficult to assess whether the survival benefit is attributable to systemic disease control or to an activity on BM in presence of an altered BBB.

Given its small lipophilic molecule, lapatinib can cross the $\mathrm{BBB}$, and drug penetration at significant levels has been demonstrated in resected $\mathrm{BM}$ of patients with $\mathrm{MBC}$ treated with lapatinib plus capecitabine, suggesting that these drugs are capable of crossing the BBB. ${ }^{18}$

The long-term disease stabilization reported in the present case, for $>13$ months, in a heavily pretreated patient with the combination of nab-paclitaxel plus trastuzumab is of note for several reasons. First, nab-paclitaxel plus trastuzumab combination, despite several previous lines of treatment, some of which were associated with known activity on BM (ie, lapatinib plus capecitabine), was the first systemic therapy not associated with CNS recurrence, avoiding the recourse to additional locoregional treatments. Moreover, this combination was associated with long extracranial stabilization with minimal neurological toxicity. The remarkably lengthy PFS reported in our case with the nab-paclitaxel plus trastuzumab combination further confirms the efficacy and the favorable toxicity profile of this promising schedule, which showed intriguing results in two phase II studies in first-line MBC, with an ORR of $42.2 \%-62.5 \%$ and a median PFS of 14.5-16.6 months. ${ }^{19,20}$ However, in these studies, patients with symptomatic BM were excluded. To date, no data were reported on a possible activity of nab-paclitaxel in the CNS. Nab-paclitaxel is a solvent-free, human albuminstabilized formulation of paclitaxel. Its peculiar formulation allows a more rapid and deeper tissue penetration and slower elimination of nab-paclitaxel compared with solvent-based paclitaxel..$^{21}$ Our case study suggests a possible activity of this combination on BMs. In fact, the schedule in the present case was the only line of therapy not associated with further CNS recurrence, avoiding new SRS treatments. This combination reported a much longer PFS ( $>13$ months) than previous lines of therapy.

In conclusion, weekly nab-paclitaxel plus trastuzumab may represent a valuable option in the treatment of HER2positive MBC with BMs after radiotherapy and is effective and associated with good toxicity profile, even in heavily pretreated patients. Therefore, this option may be considered in patients with HER2-positive MBC with BM, after recurrence with standard first-line therapy, which now is represented by the trastuzumab + pertuzumab + docetaxel combination..$^{22}$ In addition, the present case further confirms the positive impact of the use of trastuzumab beyond, as emerged in the GBG 26 trial. $^{23}$

\section{Disclosure}

The authors report no conflicts of interest in this work.

\section{References}

1. Mehta AI, Brufsky AM, Sampson JH. Therapeutic approaches for HER2positive brain metastases: circumventing the blood-brain barrier. Cancer Treat Rev. 2013;39(3):261-269.

2. Lin NU, Amiri-Kordestani L, Palmieri D, Liewehr DJ, Steeg PS. CNS metastases in breast cancer: old challenge, new frontiers. Clin Cancer Res. 2013;19(23):6404-6418.

3. Chien AJ, Rugo HS. Emerging treatment options for the management of brain metastases in patients with HER2-positive metastatic breast cancer. Breast Cancer Res Treat. 2013;137(1):1-12. 
4. Bria E, Cuppone F, Fornier M, et al. Cardiotoxicity and incidence of brain metastases after adjuvant trastuzumab for early breast cancer: the dark side of the moon? A meta-analysis of the randomized trials. Breast Cancer Res Treat. 2008;109(2):231-239.

5. Viani GA, Afonso SL, Stefano EJ, De Fendi LI, Soares FV. Adjuvant trastuzumab in the treatment of her-2-positive early breast cancer: a metaanalysis of published randomized trials. BMC Cancer. 2007;7:153.

6. Olson EM, Abdel-Rasoul M, Maly J, Wu CS, Lin NU, Shapiro CL. Incidence and risk of central nervous system metastases as site of first recurrence in patients with HER2-positive breast cancer treated with adjuvant trastuzumab. Ann Oncol. 2013;24(6):1526-1533.

7. Steeg PS, Camphausen KA, Smith QR. Brain metastases as preventive and therapeutic targets. Nat Rev Cancer. 2011;11(5):352-363.

8. Eichler AF, Chung E, Kodack DP, Loeffler JS, Fukumura D, Jain RK. The biology of brain metastases-translation to new therapies. Nat Rev Clin Oncol. 2011;8(6):344-356.

9. Lockman PR, Mittapalli RK, Taskar KS, et al. Heterogeneous blood-tumor barrier permeability determines drug efficacy in experimental brain metastases of breast cancer. Clin Cancer Res. 2010;16(23):5664-5678.

10. Yonemori K, Tsuta K, Ono M, et al. Disruption of the blood-brain barrier by brain metastases of triple-negative and basal-type breast cancer but not HER2/neu-positive breast cancer. Cancer. 2010;116(2):302-308.

11. van Vulpen M, Kal HB, Taphoorn MJ, El-Sharouni SY. Changes in blood-brain barrier permeability induced by radiotherapy: implications for timing of chemotherapy? (Review). Oncol Rep. 2002;9(4): 683-688.

12. Smith I, Procter M, Gelber RD, et al; HERA study team. 2-year follow-up of trastuzumab after adjuvant chemotherapy in HER2-positive breast cancer: a randomised controlled trial. Lancet. 2007;369(9555):29-36.

13. Romond EH, Perez EA, Bryant J, et al. Trastuzumab plus adjuvant chemotherapy for operable HER2-positive breast cancer. NEngl J Med. 2005;353(16):1673-1684.

14. Slamon DJ, Leyland-Jones B, Shak S, et al. Use of chemotherapy plus a monoclonal antibody against HER2 for metastatic breast cancer that overexpresses HER2. N Engl J Med. 2001;344(11):783-792.
15. Bartsch R, Rottenfusser A, Wenzel C, et al. Trastuzumab prolongs overall survival in patients with brain metastases from Her2 positive breast cancer. J Neurooncol. 2007;85(3):311-317.

16. Church DN, Modgil R, Guglani S, et al. Extended survival in women with brain metastases from HER2 overexpressing breast cancer. Am J Clin Oncol. 2008;31(3):250-254.

17. Park IH, Ro J, Lee KS, Nam BH, Kwon Y, Shin KH. Trastuzumab treatment beyond brain progression in HER2-positive metastatic breast cancer. Ann Oncol. 2009;20(1):56-62.

18. Morikawa A, Peereboom DM, Thorsheim HR, et al. Capecitabine and lapatinib uptake in surgically resected brain metastases from metastatic breast cancer patients: a prospective study. Neuro Oncol. 2015;17(2):289-295.

19. Mirtsching B, Cosgriff T, Harker G, Keaton M, Chidiac T, Min M. A phase II study of weekly nanoparticle albumin-bound paclitaxel with or without trastuzumab in metastatic breast cancer. Clin Breast Cancer. 2011;11(2):121-128.

20. Conlin AK, Seidman AD, Bach A, et al. Phase II trial of weekly nanoparticle albumin-bound paclitaxel with carboplatin and trastuzumab as first-line therapy for women with HER2-overexpressing metastatic breast cancer. Clin Breast Cancer. 2010;10(4):281-287.

21. Chen N, Li Y, Ye Y, Palmisano M, Chopra R, Zhou S. Pharmacokinetics and pharmacodynamics of nab-paclitaxel in patients with solid tumors: disposition kinetics and pharmacology distinct from solvent-based paclitaxel. J Clin Pharmacol. 2014;54(10):1097-1107.

22. Baselga J, Cortés J, Kim SB, et al; CLEOPATRA Study Group. Pertuzumab plus trastuzumab plus docetaxel for metastatic breast cancer. N Engl J Med. 2012;366(2):109-119.

23. von Minckwitz G, Schwedler K, Schmidt M, et al; GBG 26/BIG 03-05 study group and participating investigators. Trastuzumab beyond progression: overall survival analysis of the GBG 26/BIG 3-05 phase III study in HER2-positive breast cancer. Eur J Cancer. 2011;47(15): 2273-2281.
OncoTargets and Therapy

\section{Publish your work in this journal}

OncoTargets and Therapy is an international, peer-reviewed, open access journal focusing on the pathological basis of all cancers, potential targets for therapy and treatment protocols employed to improve the management of cancer patients. The journal also focuses on the impact of management programs and new therapeutic agents and protocols on

\section{Dovepress}

patient perspectives such as quality of life, adherence and satisfaction The manuscript management system is completely online and includes a very quick and fair peer-review system, which is all easy to use. Visit http://www.dovepress.com/testimonials.php to read real quotes from published authors. 